\title{
772.
}

\section{ON THE ANALYTICAL FORMS CALLED TREES.}

[From the American Journal of Mathematics, vol. Iv. (1881), pp. 266-268.]

IN a tree of $N$ knots, selecting any knot at pleasure as a root, the tree may be regarded as springing from this root, and it is then called a root-tree. The same tree thus presents itself in various forms as a root-tree; and if we consider the different root-trees with $N$ knots, these are not all of them distinct trees. We have thus the two questions, to find the number of root-trees with $N$ knots; and, to find the number of distinct trees with $N$ knots.

I have in my paper "On the Theory of the Analytical Forms called Trees," Phil. Mag., t. XIII. (1857), pp. 172-176, [203] given the solution of the first question; viz. if $\phi_{N}$ denotes the number of the root-trees with $N$ knots, then the successive numbers $\phi_{1}, \phi_{2}, \phi_{3}$, etc., are given by the formula

$$
\phi_{1}+x \phi_{2}+x^{2} \phi_{3}+\ldots=(1-x)^{-\phi_{1}}\left(1-x^{2}\right)^{-\phi_{2}}\left(1-x^{3}\right)^{-\phi_{3}} \ldots,
$$

viz. we thus find

$$
\begin{array}{rlcccccccccccc}
\text { suffix of } \phi & 1 & 2 & 3 & 4 & 5 & 6 & 7 & 8 & 9 & 10 & 11 & 12 & 13 \\
\hline \phi=1 & 1 & 2 & 4 & 9 & 20 & 48 & 115 & 286 & 719 & 1842 & 4766 & 12486 .
\end{array}
$$

And I have, in the paper "On the analytical forms called Trees, with application to the theory of chemical combinations," Brit. Assoc. Report, 1875, pp. 257-305, [610] also shown how by the consideration of the centre or bicentre "of length" we can obtain formulæ for the number of central and bicentral trees, that is, for the number 
of distinct trees, with $N$ knots: the numerical result obtained for the total number of distinct trees with $N$ knots is given as follows:

\begin{tabular}{|c|c|c|c|c|c|c|c|c|c|c|c|c|c|c|}
\hline No. of Knots & & 1 & 2 & 3 & 4 & 5 & 6 & & 8 & 9 & 10 & 11 & 12 & 13 \\
\hline No. of Central & rees & 1 & 0 & 1 & 1 & 2 & 3 & 7 & 12 & 27 & 55 & 127 & 284 & 682 \\
\hline " Bicentr & " & 0 & 1 & 0 & 1 & 1 & 3 & E & 11 & 20 & 51 & 108 & 267 & 619 \\
\hline tal & & 1 & 1 & 1 & 2 & 3 & 6 & & 23 & 47 & 106 & 235 & 551 & 1301 \\
\hline
\end{tabular}

But a more simple solution is obtained by the consideration of the centre or bicentre "of number." A tree of an odd number $N$ of knots has a centre of number, and a tree of an even number $N$ of knots has a centre or else a bicentre of number. To explain this notion (due to M. Camille Jordan) we consider the branches which proceed from any knot, and (excluding always this knot itself) we count the number of the knots upon the several branches; say these numbers are $\alpha, \beta, \gamma, \delta$, $\epsilon$, etc., where of course $\alpha+\beta+\gamma+\delta+\epsilon+$ etc. $=N-1$. If $N$ is even we may have, say $\alpha=\frac{1}{2} N$; and then $\beta+\gamma+\delta+\epsilon+$ etc. $=\frac{1}{2} N-1$, viz. $\alpha$ is larger by unity than the sum of the remaining numbers: the branch with $\alpha$ knots, or the number $\alpha$, is said to be "merely dominant." If $N$ be odd, we cannot of course have $\alpha=\frac{1}{2} N$, but we may have $\alpha>\frac{1}{2} N$; here $\alpha$ exceeds by 2 at least the sum of the other numbers; and the branch with $\alpha$ knots, or the number $\alpha$, is said to be "predominant." In every other case, viz. in the case where each number $\alpha$ is less than $\frac{1}{2} N$, (and where consequently the largest number $\alpha$ does not exceed the sum of the remaining numbers), the several branches, or the numbers $\alpha, \beta, \gamma$, etc., are said to be subequal. And we have the theorem. First, when $N$ is odd, there is always one knot (and only one knot) for which the branches are subequal: such knot is called the centre of number. Secondly, when $N$ is even, either there is one knot (and only one knot) for which the branches are subequal: and such knot is then called the centre of number; or else there is no such knot, but there are two adjacent knots (and no other knot) each having a merely-dominant branch: such two knots are called the bicentre of number, and each of them separately is a half-centre.

Considering now the trees with $N$ knots as springing from a centre or a bicentre of number, and writing $\psi_{N}$ for the whole number of distinct trees with $N$ knots, we readily obtain these in terms of the foregoing numbers $\phi_{1}, \phi_{2}, \phi_{3}$, etc., viz. we have

$$
\begin{aligned}
& \psi_{1}=\quad 1, \\
& \psi_{2}=\frac{1}{2} \phi_{1}\left(\phi_{1}+1\right), \\
& \psi_{3}=\quad \text { coeff. } x^{2} \text { in }(1-x)^{-\phi_{1}}, \\
& \psi_{4}=\frac{1}{2} \phi_{2}\left(\phi_{2}+1\right)+\text { coeff. } x^{3} \text { in }(1-x)^{-\phi_{1}} \\
& \psi_{5}=\quad \text { coeff. } x^{4} \text { in }(1-x)^{-\phi_{1}}\left(1-x^{2}\right)^{-\phi_{2}} \\
& \psi_{6}=\frac{1}{2} \phi_{3}\left(\phi_{3}+1\right)+\text { coeff. } x^{5} \text { in }(1-x)^{-\phi_{1}}\left(1-x^{2}\right)^{-\phi_{2}}, \\
& \psi_{7}=\quad \quad \text { coeff. } x^{6} \text { in }(1-x)^{-\phi_{1}}\left(1-x^{2}\right)^{-\phi_{2}}\left(1-x^{3}\right)^{-\phi_{3}},
\end{aligned}
$$


and so on, the law being obvious. And the formulæ are at once seen to be true. Thus for $N=6$, the formula is

$$
\begin{aligned}
\psi_{6}=\frac{1}{2} \phi_{3}\left(\phi_{3}+1\right)+\frac{1}{2} \phi_{2}\left(\phi_{2}+1\right) \cdot \phi_{1}+\phi_{2} \cdot \frac{1}{6} \phi_{1}\left(\phi_{1}+1\right)\left(\phi_{1}+2\right) \\
\\
+\frac{1}{120} \phi_{1}\left(\phi_{1}+1\right)\left(\phi_{1}+2\right)\left(\phi_{1}+3\right)\left(\phi_{1}+4\right) .
\end{aligned}
$$

We have $\phi_{3}$ root-trees with 3 knots, and by simply joining together any two of them, treating the two roots as a bicentre, we have all the bicentral trees with 6 knots: this accounts for the term $\frac{1}{2} \phi_{3}\left(\phi_{3}+1\right)$. Again, we have $\phi_{1}$ root-trees with 1 knot, $\phi_{2}$ root-trees with 2 knots; and with a given knot as centre, and the partitions $(2,2,1),(2,1,1,1),(1,1,1,1,1)$ successively, we build up the central trees of 6 knots, viz. $1^{\circ}$ we take as branches any two $\phi_{2}$ 's and any one $\phi_{1} ; 2^{\circ}$ any one $\phi_{2}$ and any three $\phi_{1}{ }^{\prime} \mathrm{s} ; 3^{\circ}$ any five $\phi_{1}$ 's; the partitions in question being all the partitions of 5 with no part greater than 2, that is, all the partitions with subequal parts. We easily obtain

$$
\begin{array}{rccccccccccccc}
\text { suffix of } \psi & 1 & 2 & 3 & 4 & 5 & 6 & 7 & 8 & 9 & 10 & 11 & 12 & 13 \\
\psi=1 & 1 & 1 & 2 & 3 & 6 & 11 & 23 & 47 & 106 & 235 & 551 & 1301
\end{array}
$$

agreeing with the results obtained by the much more complicated formulæ of the paper of 1875 . 